\title{
Electro-immuno sensors: current developments and future trends
}

\begin{abstract}
Although immunoassays have been widely used in laboratories and medical facilities for the effective detection of different proteins, their long times and costs have produced the need of developing substitute technologies. Currently, electro-immuno sensors have appeared as a solution that can be turned into point-of-care devices for diagnosis purposes. Electro-immuno sensors are biosensors that use an antibody as the recognition element and electrochemical techniques as tranducers, and have been principally developed in a disposable format. This review attempts to describe the current state-of-the-art of immune tests used in laboratories and medical facilities for the effective detection of a wide number of proteins, and how they are seemed to be migrating from chromatographic measurements to electrochemical ones. Finally, it exposes the general considerations that should be taken into account in the development of commercial electro-immuno sensors, in which concepts as the Internet of Things (IoT), cloud computing and wireless connectivity are explored.
\end{abstract}

Keywords: electro-immuno sensors, antibody-based biosensors, electrochemical transducers, hydroxyapatite powder, antibody immobilization, disposable electroimmuno sensors, redox reactions
Volume 2 Issue I - 2017

\author{
Natalia Lopez Barbosa, Crhistian Segura, \\ Johann F Osma \\ Department of Electrical \& Electronics Engineering, Universidad
} de los Andes, Colombia

Correspondence: Johann F Osma, CMUA, Department of Electrical and Electronics Engineering, Universidad de los Andes, Cra IE No 19a-40, Bogota, DC I | |7| |, Colombia, Email jf.osma43@uniandes.edu.co

Received: October 21, 2016 | Published: January 24, 2017
Abbreviations: Iot, internet of things; ELISA, enzyme-linked immuno sorbent assay; WB, western blot; IHC, immunohistochemistry; ICC, immunocytochemistry; FACS, fluorescence-activated cell sorting; IP, immuno precipitation; ELISPOT, enzyme-linked immuno spot; PSA, prostate specific antigen; IDEs, interdigitated electrodes; HPV, human papilloma virus

\section{Introduction}

Biosensors have been mostly used in the food industry, medical and biological fields, among others, where physical and chemical sensors cannot accurately measure all variables. Biosensors are usually comprised by a biological recognition element and a transducer. ${ }^{1,2}$ Among the different commercial biosensors, the most common recognition elements are DNA, ${ }^{3}$ enzymes, ${ }^{4}$ aptamers ${ }^{5,6}$ and antibodies. ${ }^{7,8}$ DNA-based biosensors detect specific analytes by their cleavage and ligation to a functionalized DNA strand. ${ }^{9}$ Enzymatic biosensors immobilize an enzyme to an electrode to facilitate the transport of electrons from the enzyme to the electrode, produced by specific reactions at the active site of the enzyme. ${ }^{2}$ Aptamers are chemically synthesized oligonucleotides ${ }^{10}$ that provide high specificity and sensitivity towards a specific target. Antibody-based biosensors recognize a specific antigen from the immobilization of a monoclonal or polyclonal antibody. ${ }^{8}$ In general, transducers vary according to the end user towards which the sensor is thought. Among others, transducers include electrochemical responses, mass changes, optical absorption or transmission, and thermal readings. ${ }^{2}$

During the last decades, several immunoassay techniques have appeared as a response to the need of having specific and sensitive tests for antigen recognition. The methods by which the recognition is perform varies from one to another, but the presence of at least one recognition antibody remains as a constant. Among others, the most common immune tests used in laboratories and medical facilities are the enzyme-linked immuno sorbent assay (ELISA), western blot (WB), immunohistochemistry (IHC), immunocytochemistry (ICC), fluorescence-activated cell sorting (FACS), immuno precipitation (IP), and enzyme-linked immuno spot (ELISPOT). Figure 1 shows a general diagram of these immunoassays.

ELISA is a widely used technique for the recognition of antibodies' antigens within a sample by means of the biochemical recognition of an enzyme. ${ }^{11}$ In this technique, specific antibodies are conjugated to an enzyme that catalyzes a colorimetric molecule. Spectrophotometric measurements are used to determine the antigen concentration in the sample. ELISA tests can be performed in a qualitative way, in which the results are positive or negative by statistical methods; or quantitative, in which a standard curve from spectrophotometric measurements can be used to quantify the concentration of the antigen. ${ }^{12}$

WB is a technique for the detection of specific proteins in a sample through the electrophoretic transfer from a separating gel to a blotting matrix. ${ }^{13} \mathrm{WB}$ uses available monoclonal or polyclonal antibodies for the detection of specific polypeptides. This allows the optimal combination of the high resolution from electrophoresis and the high sensitivity from immunoassays. ${ }^{14}$ Antibodies are labeled so that the presence of a particular protein can be detected by means of chemiluminescence.

As performed by WB technique, IHC is a method for specific protein recognition through antibody binding. However, in IHC, protein expression can be characterized directly from the intact tissue ${ }^{15}$ For this reason, this technique has been used in the diagnosis of tumors ${ }^{16}$ and carcinomas, ${ }^{17}$ where proteins play a decisive role. Similarly, ICC is used to study the sub cellular distribution of proteins through fluorescent labeled antibodies. ${ }^{18}$ Contrarily to IHC, ICC has shown to work during in vivo measurements of amyloid fibrils by human lysozyme. ${ }^{19}$ However, most successful ICC applications use antibodies binded to oligonucleotides for in situ measurements. ${ }^{20,21}$

FACS is a technique in which the quantification of a fluorescent signal is used to separate cells by a particular physical characteristic (e.g. cell-size, fluorescence intensity). ${ }^{22}$ This method has been 
primarily used in research experiments, although Breser et al..$^{23}$ has shown its potential for detecting prostate inflammation in autoimmune prostatitis. IP technique enables the isolation and purification of complexed and individual proteins by immobilizing antibodies onto solid substrates. ${ }^{24}$ This technique can be used to analyze the chromatin of a particular cell, in which DNA/protein interactions are observed, ${ }^{25}$ and can be used to determine the binding sites of chromosomal proteins. This correlation technique between proteins and gene expression has contributed to the growth of bioinformatics libraries that help scientists to understand the effect of specific proteins in living organisms.

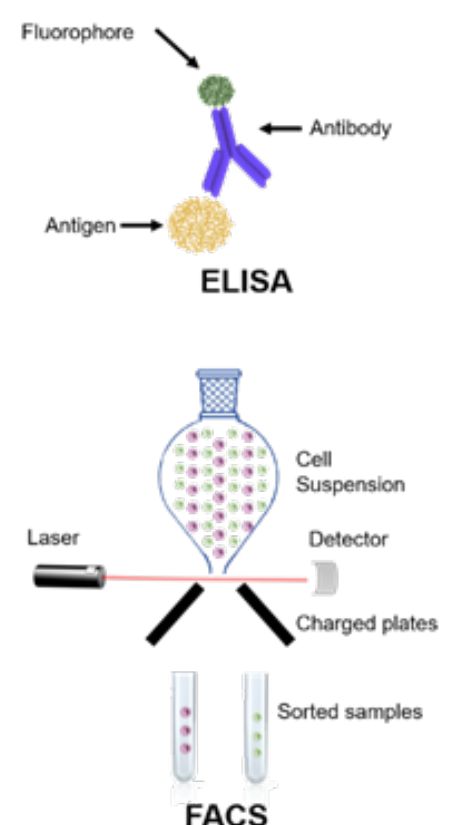

FACS
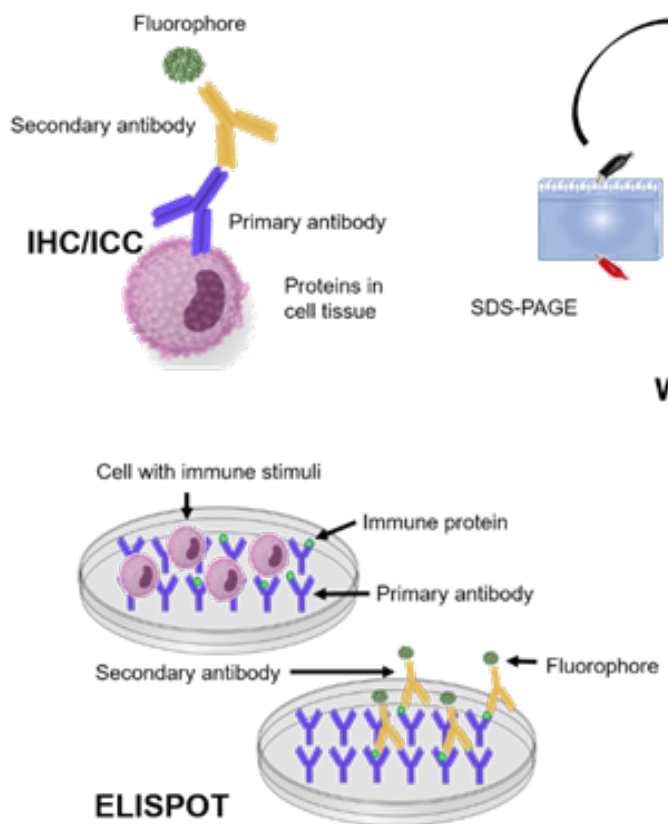

WB
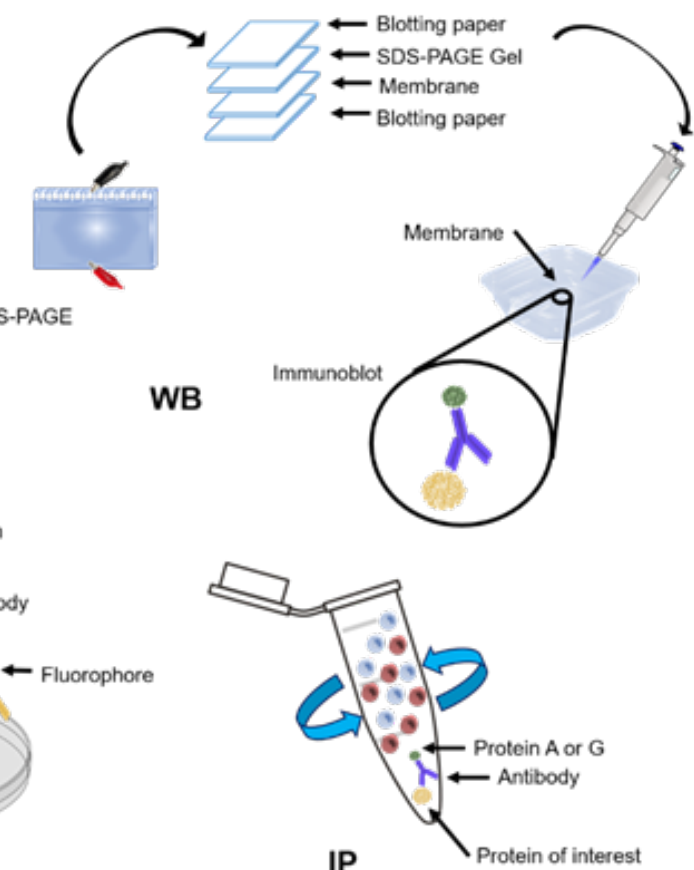

Figure I Diagram of the different immunoassays.

ELISPOT assay was created for the detection of antigenspecific immune response in single cells. ${ }^{26}$ Nowadays, ELISPOT is used for a variety of applications like monitoring the response of immunotherapeutic treatment in cancer patients, ${ }^{27-29}$ and the specific immune response in patients with infectious, ${ }^{30}$ neoplastic ${ }^{31}$ and autoimmune ${ }^{32}$ diseases. All the immunoassays mentioned above have been part of the usual protein quantification and detection techniques. Moreover, all of them are based in recognition by chromatographic principles, require specific technologies, and trained individuals to carry out the test. This has created difficulties in the integration of the current immunoassay techniques for point-of-care devices, where non-trained individuals should be able to carry out the tests and get reliable results. ${ }^{2}$ In response, protein recognition by electrochemical techniques has emerged and has been integrated in what is known as electro-immuno sensors.

Electro-immuno sensors are antibody-based biosensors that use electrochemical transducers. The charge transport capacity of the electrodes of these sensors can be measured by cyclic voltammetry (CV) or as a change in the electrical impedance by electrochemical impedance spectroscopy (EIS). These sensors have been recently used in a variety of point-of-care detection devices, and have shown to be promising for liver function, ${ }^{33}$ wound infection biomarkers ${ }^{34}$ and cancer biomarkers ${ }^{35,36}$ detection. This review attempts to describe the migration of electrochemical techniques such as CV and EIS from characterization to point-of-care detection techniques. Finally, it exposes the general considerations that should be taken into account in the development of commercial point-of-care devices based on electro-immuno sensors, in which complementary concepts as the
Internet of Things (IoT), cloud computing and wireless connectivity are explored.

\section{Cyclic voltammetry (CV) and electrochemical impedance spectroscopy (EIS)}

$\mathrm{CV}$ is an electrochemical technique that allows the study redox reactions at the electrode solution interfaces. The procedure uses a reference, a working, and a counter electrode to measure the current that is generated in an electrochemical cell when there is an excess of voltage from that predicted by Nernst equation. ${ }^{37}$ The conditions under which the $\mathrm{CV}$ is performed are usually controlled with a potentiostat. A periodic potential is applied between the working and reference electrode to produce redox reactions at the working electrode. The counter electrode is used to avoid a current flow between the working and reference electrode that might change the applied voltage. ${ }^{38}$ A measurement of the current generated between the counter and working electrode in relation with the potential applied between the reference and working electrode gives information of the oxidation and reduction of the analyte.

EIS is a technique that studies an electrochemical system by observing its behavior as an idealized model circuit with discrete electrical components. EIS applies an alternating potential between two electrodes and measures the current generated through the sample. The potential is applied at different frequencies to generate the impedance spectrum of the sample. Contrary to metal samples, where the charge carriers are electrons, in biological samples, ions constitute the main charge carriers and thus, the conductivity is dependent on their concentration and diffusion coefficients. ${ }^{39}$ 
CV and EIS techniques have been commonly used to characterize the electrical and redox nature of different types of samples. Nevertheless, they have recently become a method for characterizing biological samples and understanding the behavior of biological systems. For instance, Chang et al. ${ }^{40}$ used fast-scan CV to monitor the presence of adenosine in patients during deep brain stimulation. $\mathrm{CV}$ allowed them to correlate the presence of microthalamotomy with neurochemical changes when introducing the stimulating electrode. Similarly, Yun et al. ${ }^{41}$ fabricated an EIS measurement system at the end of a hypodermic needle to differentiate between healthy and damaged tissues prior to biopsy. CV and EIS techniques have shown to be easily integrated in lab-on-a-chip devices ${ }^{2,42}$ for point-of-care applications based on electro-immuno sensors.

\section{Electro-immuno sensors}

In general terms, all different antigen recognition techniques used in laboratories and medical facilities can be used as a base for electro-immuno sensors. However, the modified electrode technique, where the electrode serves as the support material for the antibody immobilization, has been widely explored ${ }^{43}$ During the measurement, electrochemical techniques play a fundamental role and can be easily adapted for multiplex immunoassays. ${ }^{44-46}$ This technique is illustrated in (Figure 2), where the presence of a binded antigen produces an electrochemical change.

$\Delta$ Detection with and without antigen

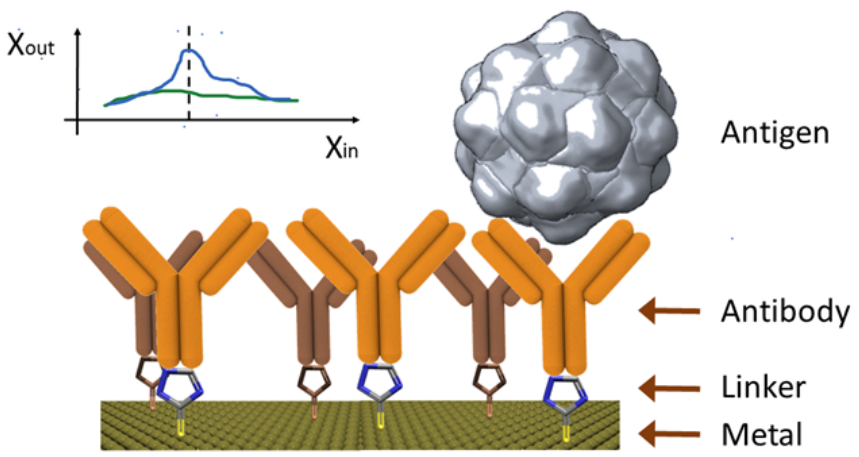

Figure 2 Most common configuration of electro-immuno sensor devices and their response with and without the presence of the antigen.

In theory, any electrode can be adapted for the immobilization of antibodies on its surface. Nevertheless, screen printed electrodes have been preferred due to their low cost, easy manufacture and suitability for mass production..$^{47}$ Although there is a preference for disposable electro-immuno sensors, ${ }^{48-50}$ there is a growing trend of surface renewal for reusable purposes. ${ }^{51}$ However, the latter involves long renewal times, which increases the response time of the system.

In order to adapt the electrode for further immobilization, nanomaterials such as gold nanoparticles ${ }^{52,53}$ and hydroxyapatite powder, ${ }^{54}$ membranes with ion-exchange sites, ${ }^{55}$ and polymeric films, ${ }^{56}$ are commonly used. One possible way to make the electro-immuno sensor semi-reusable is by attaching the antibodies to magnetic nanoparticles prior to their immobilization on the electrode surface. In this way, the deposition of the sensible layer can be controlled by an external magnetic field..$^{57}$

Currently, there are many different manufacture methods to obtain a functional electro-immuno sensor. For instance, Duangkaew et al. ${ }^{58}$ showed a novel method to amplify the signal of a biosensor that uses a prostate specific antigen (PSA) as the recognition element. The authors developed a sandwich based biosensor in which PSA is placed between two antibodies. One of them is attached to a substrate while the other is attached to a matrix of gold nanoparticles (AuNP). The presence of AuNP facilitated the growth of gold spikes increasing the area-to-volume ratio. This increased the output current of the sensor when tested by anodic stripping analysis.

Interdigitated electrodes (IDEs) are commonly used when performing electro-immuno sensors based on EIS. Singh et al. ${ }^{59} \mathrm{Yu}$ et al. ${ }^{60}$ and Zhang et al. ${ }^{61}$ developed electro-immuno sensors with IDEs by using rectangular arrays for measuring the presence of Ricin. Authors measured the impedance variation by CV and EIS on the IDEs. Hsieh et al. ${ }^{62}$ assembled a biosensor with ring-shaped IDEs to promote an uniform distribution and an efficient immobilization of glycated hemoglobin (HbAlc).

In the same way, Osma et $a 1 .{ }^{46}$ designed a fabrication process of an electro-immuno sensor based on the immobilization of a monoclonal Antibody (mAb) 5051 on gold IDEs for the detection of the Human Papilloma Virus (HPV) 16. In this case, mAb 5051 was attached to a gold layer through thiols. CV measurements allowed the effective detection of HPV 16. A similar process was developed by Monerris et al. ${ }^{63}$ where an electro-immuno sensor based on $\mathrm{CV}$ measurements was used to sense the presence of estrone hormone in water samples. This was attained by immobilizing a sheep anti estrone $\mathrm{mAb}$ on a layer of AuNPs.

Hou et al. ${ }^{64}$ and Freitas et al., ${ }^{65}$ fabricated electro-immuno sensors that measured through EIS and CV for the detection of microcystinLR and Cry1 Ab, respectively. Similarly, Moschou et al. ${ }^{66}$ used double layers printed circuit boards (PCBs) to fabricate electrodes, reducing manufacturing time and costs. On the copper electrodes, gold and silver layers were coated for the further antibody immobilization. Measurements were performed through CV techniques. The authors compared the electro-immuno sensor with an ELISA immunoassay, showing that it was possible to detect $\mathrm{TMB} / \mathrm{H}_{2} \mathrm{O}_{2}$ through electrochemical methods.

\section{Future trends}

Electro-immuno sensors have appeared as a novel trending solution for specific and high sensitive biosensors. It has been proven that using immune detection results in a feasible solution for specific protein detection in low concentrated and low volume samples. This constitutes a promising technique for point-of-care devices, in which a low sample and reagent volume requirements, easy integration and rapid response are desired. ${ }^{2}$ However, current electro-immuno sensors are still an emerging trend, and so, several aspects should be considered in order to achieve a commercial and user friendly product.

For instance, current electro-immuno sensors should consider their communication with front-end technology such as smart phones and computers, so that there is an easy monitoring and data storage. User-friendly applications must be developed to receive and process information from the sensor device. Similarly, in order to migrate electro-immuno sensors from laboratory to industrial products, their manufacture process needs to be scalable and easy to automatize. Either if they are used as point-of-care devices or as laboratory instrumentation, they should contain an user-friendly interface, which is usually approached with a sample-to-answer format. ${ }^{67}$ In addition, independent rechargeable energy sources should be considered if the electro-immuno sensor is meant to be portable. Finally, concepts as the IoT and cloud computing should be integrated with the sensors circuitry. 


\section{Acknowledgements}

None.

\section{Conflict of interest}

The author declares no conflict of interest.

\section{References}

1. Mehrotra P. Biosensors and their applications - A review. J Oral Biol Craniofacial Res. 2016;6(2):153-159.

2. Lopez Barbosa N, Gamarra JD, Osma JF. The future point-of-care detection of disease and its data capture and handling. Anal Bioanal Chem.2016;408(11):2827-2837.

3. Gao L, Lian C, Zhou Y, et al. Graphene oxide-DNA based sensors. Biosens Bioelectron. 2014;60:22-29.

4. Wang J. Electrochemical glucose biosensors. Chem Rev. 2008;108(2):814-825.

5. Li H, Bai X, Wang N, et al. Aptamer-based micro cantilever biosensor for ultrasensitive detection of tumor marker nucleolin. Talanta. 2016;146:727-731.

6. Famulok M, Mayer G. Aptamer modules as sensors and detectors. Acc Chem Res. 2011;44(12):1349-1358.

7. Vachali PP, Li B, Bartschi A, et al. Surface plasmon resonance (SPR)based biosensor technology for the quantitative characterization of protein-carotenoid interactions. Arch Biochem Biophys. 2015;572:66-72.

8. Skottrup PD, Nicolaisen M, Justesen AF. Towards on-site pathogen detection using antibody-based sensors. Biosens Bioelectron. 2008;24(3):339-348.

9. Nagraj N. Development of functional DNA-based sensors and investigations into their mechanism. 2010. 198 p.

10. Acquah C, Danquah MK, Yon JLS, et al. A review on immobilised aptamers for high throughput biomolecular detection and screening. Anal Chim Acta. 2015;888:10-18.

11. Asensio L, González I, García T, et al. Determination of food authenticity by enzyme-linked immuno sorbent assay (ELISA). Food Control. 2008;19:1-8.

12. Goldsby RA, Kindt TJ, Osborne BA, et al. Enzyme-linked immuno sorbent assay. Immunology. 2003;5:148-150.

13. Towbin H, Staehelin T, Gordon J. Electrophoretic transfer of proteins from polyacrylamide gels to nitrocellulose sheets: procedure and some applications. Proc Natl Acad Sci USA. 1979;76(9):4350-4354.

14. Ghirardello A, Bendo R, Rampudda ME, et al. Commercial blot assays in the diagnosis of systemic rheumatic diseases. Autoimmun Rev. 2009;8(8):645-649.

15. Schofield DJ, Lewis AR, Austin MJ. Genetic methods of antibody generation and their use in immunohistochemistry. Methods. 2014;70(1):20-27.

16. Longo FR, Angelico G, Spadola S, et al. Immunohistochemistry as potential diagnostic pitfall in the most common solid tumors of children and adolescents. Acta Histochem. 2015;117(4-5):397-414.

17. Rebecca D Chernock. Immunohistochemistry of thyroid gland carcinomas: clinical utility and diagnostic pitfalls. Diagnostic Histopathol. 2016;22(5):184-190.

18. Tanke HJ, Dirks RW, Raap T. FISH and immunocytochemistry: towards visualising single target molecules in living cells. Curr Opin Biotechnol. 2005;16(1):49-54.
19. Dumoulin M, Last AM, Desmyter A, et al. A camelid antibody fragment inhibits the formation of amyloid fibrils by human lysozyme. Nature. 2003;424(6950):783-788.

20. Gusev Y, Sparkowski J, Raghunathan A, et al. Rolling circle amplification: a new approach to increase sensitivity for immunohistochemistry and flow cytometry. Am J Pathol. 2001;159(1):63-69.

21. Schweitzer B, Roberts S, Grimwade B, et al. Multiplexed protein profiling on microarrays by rolling-circle amplification. Nat Biotech. 2002;20(4):359-365.

22. Herzenberg LA, De Rosa SC, Herzenberg LA. Monoclonal antibodies and the FACS: complementary tools for immunobiology and medicine. Immunol Today. 2000;21(8):383-390.

23. Breser ML, Motrich RD, Sanchez LR, et al. Chronic pelvic pain development and prostate inflammation in strains of mice with different susceptibility to experimental autoimmune prostatitis. Prostate. 2016;77(1):94-104

24. Li Y, Collins M, An J, et al. Immuno precipitation and mass spectrometry defines an extensive RBM45 protein-protein interaction network. Brain Res. 2016;1647:79-93.

25. Orlando V. Mapping chromosomal proteins in vivo by formaldehydecross linked-chromatin immuno precipitation. Trends Biochem Sci. 2000;25(3):99-104.

26. Czerkinsky CC, Tarkowski A, Nilsson L- $\AA$, et al. Reverse enzyme-linked immuno spot assay (RELISPOT) for the detection of cells secreting immuno reactive substances. J Immunol Methods. 1984;72(2):489-496.

27. Van der Zee AGJ, Melief CJ, Van der Burg SH, et al. Immunization with a P53 synthetic long peptide vaccine induces P53-specific immune responses in ovarian cancer patients, a phase II trial. Int $J$ cancer. 2009;125(9):2104-2113.

28. Palmer DH, Midgley RS, Mirza N, et al. A phase II study of adoptive immunotherapy using dendritic cells pulsed with tumor lysate in patients with hepatocellular carcinoma. Hepatology. 2009;49(1):124-132.

29. Schuetz F, Ehlert K, Ge Y, et al. Treatment of advanced metastasized breast cancer with bone marrow-derived tumour-reactive memory T cells: a pilot clinical study. Cancer Immunol Immunother. 2008;58(6):887-900.

30. Walker JM, Slifka MK. Longevity of T-Cell Memory following Acute Viral Infection BT - Memory T Cells. Zanetti M, et al. editors. New York, USA: Springer; 2010. p. 96-107

31. Kabingu E, Oseroff AR, Wilding GE, et al. Enhanced systemic immune reactivity to a Basal cell carcinoma associated antigen following photodynamic therapy. Clin Cancer Res. 2009;15(13):4460-4466.

32. Zanone MM, Favaro E, Quadri R, et al. Association of cytomegalovirus infections with recurrence of humoral and cellular autoimmunity to islet autoantigens and of type 1 diabetes in a pancreas transplanted patient. Transpl Int. 2010;23(3):333-337.

33. Chuang YH, Chang YT, Liu KL, et al. Electrical impedimetric biosensors for liver function detection. Biosens Bioelectron. 2011;28(1):368-372.

34. Ciani I, Schulze H, Corrigan DK, et al. Development of immuno sensors for direct detection of three wound infection biomarkers at point of care using electrochemical impedance spectroscopy. Biosens Bioelectron. 2012;31(1):413-418.

35. Elshafey R, Tlili C, Abulrob A, et al. Label-free impedimetric immunosensor for ultrasensitive detection of cancer marker Murine double minute 2 in brain tissue. Biosens Bioelectron. 2013;39(1):220 225 .

36. Li H, He J, Li S, et al. Electrochemical immunosensor with N-doped graphene-modified electrode for label-free detection of the breast cancer biomarker CA 15-3. Biosens Bioelectron. 2013;43:25-29. 
37. Bilal S. Cyclic Voltammetry BT - Encyclopedia of Applied Electrochemistry. In: Kreysa G, Ota K, et al. editors. New York, USA: Sprineger; 2014. p. 285-289.

38. Borchert H. Cyclic Voltammetry BT - Solar Cells Based on Colloidal Nanocrystals. In: Borchert H editors. Switzerland: Springer International Publishing; 2014. p. 111-117.

39. Azzarello E, Masi E, Mancuso S. Electrochemical Impedance Spectroscopy BT - Plant Electrophysiology: Methods and Cell Electrophysiology. In: Volkov AG editors. USA: Springer Berlin Heidelberg; 2012. p. 205-223

40. Chang SY, Kim I, Marsh MP, et al. Wireless fast-scan cyclic voltammetry to monitor adenosine in patients with essential tremor during deep brain stimulation. Mayo Clin Proc. 2012;87(8):760-765.

41. Yun J, Kang G, Park Y, et al. Electrochemical impedance spectroscopy with interdigitated electrodes at the end of hypodermic needle for depth profiling of biotissues. Sensors Actuators B Chem. 2016;237:984-991.

42. Yakdi NE, Huet F, Ngo K.Detection and sizing of single droplets flowing in a lab-on-a-chip device by measuring impedance fluctuations. Sensors and Actuators B Chem. 2016;236:794-804.

43. Hartwell SK, Grudpan K. Flow based immuno/bioassay and trends in micro-immuno/biosensors. Microchim Acta. 2010;169(3-4):201-220.

44. Liu Y, Gyurcsányi RE, Jágerszki G, et al. Microfabricated amperometric cells for multi component analysis. Electroanalysis. 2009;21(1718):1944-1954.

45. Lai G, Yan F, Ju H. Dual signal amplification of glucose oxidasefunctionalized nano composites as a trace label for ultrasensitive simultaneous multiplexed electrochemical detection of tumor markers Anal Chem. 2009;81(23):9730-9736.

46. Urrego LF, Lopez DI, Ramirez KA, et al. Biomicrosystem design and fabrication for the human papilloma virus 16 detection. Sensors Actuators, B Chem. 2015;207:97-104.

47. Gao Q, Ma Y, Cheng Z, et al. Flow injection electrochemical enzyme immunoassay based on the use of an immunoelectrode strip integrate immunosorbent layer and a screen-printed carbon electrode. Anal Chim Acta. 2003;488(1):61-70.

48. Killard AJ, Zhang S, Zhao H, et al. Development of an electrochemical flow injection immunoassay (FIIA) for the real-time monitoring of biospecific interactions. Anal Chim Acta. 1999;400(1-3):109-119.

49. Chen Z, Fang C, Wang H, et al. A disposable electrochemical immunofiltration test strip for rapid detection of $\alpha$-fetoprotein. Sensors Actuators B Chem. 2009;141:436-440.

50. Viswanathan S, Rani C, Anand AV, et al. Disposable electrochemical immunosensor for carcinoembryonic antigen using ferrocene liposomes and MWCNT screen-printed electrode. Biosens Bioelectron 2009;24(7):1984-1989.

51. Marquette CA, Blum LJ. Electrochemiluminescence of luminol for 2, 4-D optical immunosensing in a flow injection analysis system. Sensors Actuators B Chem. 1998;51:100-106.

52. Wang L, Jia X, Zhou Y, et al. Sandwich-type amperometric immunosensor for human immunoglobulin $\mathrm{G}$ using antibody-adsorbed $\mathrm{Au} / \mathrm{SiO} 2$ nanoparticles. Microchim Acta. 2010;168(3):245-251.
53. Liang W, Yi W, Li S, et al. A novel, label-free immunosensor for the detection of $\alpha$-fetoprotein using functionalised gold nanoparticles. Clin Biochem. 2009;42(15):1524-1530.

54. Yin $\mathrm{H}$, Zhou Y, Ai S, et al. Electrochemical oxidative determination of 4-nitrophenol based on a glassy carbon electrode modified with a hydroxyapatite nanopowder. Microchim Acta. 2010;169(1):87-92.

55. Elnemma EM, Hamada MA. Plastic membrane electrodes for the potentiometric determination of codeine in pharmaceutical preparations. Microchim Acta. 1997;126(1):147-151.

56. Giroud F, Gorgy K, Gondran C, et al. Impedimetric immunosensor based on a polypyrrole- antibiotic model film for the label-free picomolar detection of ciprofloxacin. Anal Chem. 2009;81(20):8405-8409.

57. Fu X, Wang J, Li N, et al. Label-free electrochemical immunoassay of carcinoembryonic antigen in human serum using magnetic nanorods as sensing probes. Microchim Acta. 2009;165:437-442.

58. Duangkaew P, Wutikhun T, Laocharoensuk R. Triple signal amplification strategy based on size and shape transformation of ultrasmall sub-10 nm gold nanoparticles tag towards sensitivity improvement of electrochemical immuno sensors. Sensors Actuators, B Chem. 2017;239:430-437.

59. Singh A, Pasha SK, Manickam P, et al. Single-domain antibody based thermally stable electrochemical immunosensor. Biosens Bioelectron. 2016;83:162-168.

60. Yu X, Esanu M, Mackay S, et al. An impedance detection circuit for applications in a portable biosensor system. Circuits and Systems (ISCAS). 2016. p. 1518-1521

61. Zhang D, Jiang J, Chen J, et al. Smartphone-based portable biosensing system using impedance measurement with printed electrodes for 2,4,6-trinitrotoluene (TNT) detection. Biosens Bioelectron. 2015;70:8188

62. Hsieh KM, Lan KC, Hu WL, et al. Glycated hemoglobin (HbA1c) affinity biosensors with ring-shaped interdigital electrodes on impedance measurement. Biosens Bioelectron. 2013;49:450-456.

63. Monerris MJ, D Eramo F, Arévalo FJ, et al. Electrochemical immunosensor based on gold nanoparticles deposited on a conductive polymer to determine estrone in water samples. Microchem J. 2016;129:71-77.

64. Hou L, Ding Y, Zhang L, et al. An ultrasensitive competitive immuno sensor for impedimetric detection of microcystin-LR via antibodyconjugated enzymatic bio catalytic precipitation. Sensors and Actuators $B$ Chem. 2016;233:63-70.

65. Freitas M, Correr W, Cancino-Bernardi J, et al. Impedimetric immunosensors for the detection of Cry1 Ab protein from genetically modified maize seeds. Sensors and Actuators B Chem. 2016;237:702709.

66. Moschou D, Greathead L, Pantelidis P, et al. Amperometric IFN- $\gamma$ immunosensors with commercially fabricated PCB sensing electrodes. Biosens Bioelectron. 2016;86:805-810.

67. Jung W, Han J, Choi JW, et al. Point-of-care testing (POCT) diagnostic systems using microfluidic lab-on-a-chip technologies. Journal Microelectron Engineering. 2015;132:46-57. 